\title{
On Parameter Identification for Ordinary Differential Equations
}

\section{U. Tadtenhahin}

Es werden Möglichkeiten der regụlarisierten Lösung von Identifikationsproblemen bei gewöhnlichen Differentialgleichungen für den Fall meßfehlerbehafteter Beobachtungsdaten untersucht. Zur Minimierung der regularisierenden Funktionale werden unter. Benutzung des Konzepts der adjungierten Aufgaben sowohl das Gradientenverfahren als auch das GauB.NewtonVerfahren diskutiert. Eine Anwendung auf eine spezielle inverse Aufgabe der Untersuchung von Untergrundgasspeichern vom Aquifertyp vervollständigt die Arbeit.

Обсуждаются возможности регуляризации решения задач идентификации для обычных дифференциальных уравнений в случае цанных солержащих опибқи измерения. Для минимизации регуляризирующего функционала при использовании концепции сопряженной задачи обсуждаетси применение градиентного метода и метода ГауссаНьютона. Дополняет работу применение этой метоцики к решению одной специальной обратноп задачи исследования подземных газөвых хранилищ водного типа.

The regularized solution of identification problems in ordinary differential equations is investigated when the data are noisy. For minimizing the occurring regularization functionals the gradient method and the Gauss-Newton method are examined by exploiting the concept of adjoint equations. An application to a particular inverse problem arising in the study of water movement about a gas-storage reservoir of aquifer type completes the paper.

\section{Introduction}

There has been increasing interest in the identification of parameters in nonlinear ordinary differential equations during the past years. In this paper we are going to discuss particular methods of regularized identification using the concept of adjoint equations. Let us consider the initial value problem

$$
\dot{x}(t)=f(t, x, a), \quad x(0)=x_{0},
$$

where

$$
x(t)=\left(\begin{array}{c}
x_{1}(t) \\
\vdots \\
x_{n}(t)
\end{array}\right), \quad a=\left(\begin{array}{c}
a_{1} \\
\vdots \\
a_{m}
\end{array}\right), \quad f(t, x, a)=\left(\begin{array}{c}
f_{1}(t, x, a) \\
\vdots \\
f_{n}(t, x, a)
\end{array}\right) .
$$

When the data are noisy the regularized parameter identification problem associated with (1.1) can be formulated as follows:

Given noisy observations $z(t)=x(t)+\delta(t)(0 \leqq t \leqq T)$ of the state $x(t)(0 \leqq t \leqq T)$, determine the parameter vector $a \in \mathbf{R}^{m}$ by minimizing the functional

$$
J_{\alpha}(a)=\frac{1}{2}\|x-z\|_{z^{2}}+\frac{\alpha}{2}\|a-\bar{a}\|_{R^{m}}^{2}
$$


over all vectors $a$ of a set $A_{\mathrm{ad}} \subset R^{m}$. Here $x=x_{a}$ designates the solution of the initial value problem (1.1) according to $a \in \mathbf{R}^{m}$.

The open subset $A_{\text {ad }} \subset \mathbf{R}^{m}$ denotes the set of physically admissible parameters $a$ for which (1.1) has a unique solution $x_{a} \in X=\left(C^{1}[0, T]\right)^{n}$. Moreover, $Z=\left(L_{2}[0, T]\right)^{n}$ denotes the space of observations, $\bar{a} \in A_{\mathrm{ad}}$ is a suitable estimate of the unknown parameters $a$ and $\alpha$ denotes the regularization parameter, which must be chosen appropriately (see e.g. [3]). A number of important identification problems in chemistry (cf. $[1,6]$ ) and other sciences fall within the above framework. An application to the modelling of gas-storage reservoirs of aquifer type is examined in Chapter 3.

Throughout this paper, the norms $\|\cdot\|_{Z}$ and $\|\cdot\|_{R^{m}}$ are assumed to be'scaled in order to equilibrate the variables. Thus, we will use the norms

$$
\|x\|_{z^{2}}=\sum_{k=1}^{n} \lambda_{k} \int_{0}^{T} x_{k}^{2}(t) d t \quad \text { and } \quad\|a\|_{R^{m}}^{2}=\sum_{k=1}^{m} \dot{\varphi}_{k} a_{k}^{2}
$$

In this context we introduce a couple of positive definite diagonal matrices

$$
\Lambda=\operatorname{diag}\left(\dot{\lambda}_{k}\right)_{1}{ }^{n} \quad \text { and } \Phi=\operatorname{diag}\left(\varphi_{k}\right)_{1}{ }^{m} .
$$

By $(\cdot, \cdot)_{Z}$ and $(\cdot, \cdot)_{R^{m}}$ we will denote the inner products in $Z$ and $\mathbf{R}^{m}$ :

$$
(x, y)_{z}=\sum_{k=1}^{n} \int_{0}^{T} x_{k}(t) y_{k}(t) d t \quad \cdots \quad \text { and } \quad(a, b)_{R^{m}}=\sum_{k=1}^{m} a_{k} b_{k}
$$

The symbols $e_{i}$ express the unit vectors in $\mathbf{R}^{m}$.

\section{Solution of the minimization problem}

In the sequel we will discuss the gradient method and the Gauss-Newton method for the numerical solution of the minimization problem (1:2). For both methods the gradient $\operatorname{grad} J_{\alpha}$,

$$
J_{\alpha}^{\prime}(a) \cdot h=\left(\operatorname{grad} J_{\alpha}(a), h\right)_{R^{m}}=\lim _{t \rightarrow 0} \frac{J_{a}(a+t h)-J_{\alpha}(a)}{t} \quad\left(h \in \mathbf{R}^{m}\right)
$$

is needed. Ijet us assume that

(A 1) for all $a \in A_{\text {ad }}$ the system (1.1) has a uniciue solution $x \in X$;

(A 2) . the transformation $H:(a, x) \in A_{\mathrm{ad}} \times X \rightarrow(\dot{x}-f(t, x, a), x(0)) \in \mathfrak{B}$, $\mathfrak{Q}=(C[0, T])^{n} \times \mathbf{R}^{1}$, is of $C\left(A_{\mathrm{ad}} \times X, \mathfrak{\mathcal { Q }}\right)$, i.e. $H$ maps continuously differentiable from $A_{\text {ad }} \times X$ into $\$$

(A 3) for every $(a, x) \in A_{\mathrm{ad}} \times X$ the mapping $H_{x}{ }^{\prime}(a, x)$ is a linear homeomorphism of $X$ onto $\mathfrak{Q}$.

Furthermore, let us introduce the notations

$$
F_{x}^{\prime}=\left(\begin{array}{ccc}
\frac{\partial f_{1}}{\partial x_{1}} & \cdots & \frac{\partial f_{1}}{\partial x_{n}} \\
\vdots & & \vdots \\
\frac{\partial f_{n}}{\partial x_{1}} & \cdots & \frac{\partial f_{n}}{\partial x_{n}}
\end{array}\right) \quad \text { and } \quad F_{a}^{\prime}=\left(\begin{array}{ccc}
\frac{\partial f_{1}}{\partial a_{1}} & \ldots & \frac{\partial f_{1}}{\partial a_{m}} \\
\vdots & & \vdots \\
\frac{\partial f_{n}}{\partial a_{1}} & \cdots & \frac{\partial f_{n}}{\partial a_{m}}
\end{array}\right)
$$

Then we can prove the following result. 
Theorem 1: Let the assumptions (A 1)-(A 3) be fulfilled. Then

(i) the functional (1.2) is of $C\left(A_{\mathrm{ad}}, \mathbf{R}^{1}\right)$, i.e. $J_{\alpha}$ maps continuously differentiable from $A_{\text {ad }}$ into $\mathbf{R}^{\mathbf{1}}$;

(ii) the gradient $\operatorname{grad} J_{a}(a) \in \mathbf{R}^{m}$ is given by

$$
\operatorname{grad} J_{\alpha}(a)=\sum_{i=1}^{m} e_{i} \int_{0}^{T}\left(y, F_{a}^{\prime} e_{i}\right)_{\mathbf{R}^{n}} d t+\alpha \Phi(a-\bar{a}),
$$

where $y$ is the solution of the adjoint system

$$
L^{*} y:=-\dot{y}(t)-F_{x}{ }^{*}(t) \dot{y}(t)=A(x(t)-z(t)), \quad y(T)=0,
$$

and $x$ is the solution of (1.1).

Proof: From the Implicit Function Theorem (cf. [10]) we obtain that under the assumptions (A 1)-(A 3) the implicit function $G: a \in A_{\mathrm{ad}} \rightarrow x \in X$ is of $C\left(A_{\mathrm{ad}}, \mathrm{X}\right)$. Hence, $x-z$ is of $C\left(A_{\text {ad }}, Z\right)$. It follows that $J_{a}$ is of $C\left(A_{\mathrm{ad}}, \mathbf{R}^{1}\right)$. Now for an arbitrary variation $\delta a \in \mathbf{R}^{m}$ one can derive

$$
J_{\alpha}^{\prime}(a) \cdot \delta a=(\Lambda(x-z), \delta x)_{z}+\alpha(\Phi(a-\bar{a}), \delta a)_{\mathbf{R}_{1}^{m}} .
$$

Furthermore, from (1.1) we get

$$
L \delta x:=\delta \dot{x}(t)-F_{x}^{\prime} \delta x(t)=F_{a}^{\prime} \delta a, \quad \dot{\delta} x(0)=0 .
$$

Using the initial conditions $y(T)=0$ and $\delta x(0)=0$, partial integration. yields

$$
(y, L \delta x)_{z}=\left(y, \delta \dot{x}-F_{x}{ }^{\prime} \delta x\right)_{z}=-\left(\dot{y}+F_{x}{ }^{*} y, \delta x\right)_{z}=\left(L^{*} y, \delta x\right)_{z}
$$

Now from (2.3), using (2.2), (2.5) and (2.4) we establish

$$
\begin{aligned}
J_{a}^{\prime}(a) \cdot \delta a & =\left(L^{*} y, \delta x\right)_{z}+\alpha(\Phi(a-\bar{a}), \delta a)_{\mathbf{R}^{m}}=(y, L \delta x)_{z}+\alpha(\Phi(a-\bar{a}), \delta a)_{\mathbf{R}^{m}} \\
& =\left(y, F_{a}^{\prime} \delta a\right)_{z}+\alpha(\Phi(a-\bar{a}), \delta a)_{\mathbf{R}^{m}} \\
& =\left(F_{a}^{\prime *} y, \delta a\right)_{z}+\alpha(\Phi(a-\bar{a}), \delta a)_{\mathbf{R}^{m}} \\
& =\left(\sum_{i=1}^{m} e_{i} \int_{0}^{T}\left(F_{a}^{\prime *} y, e_{i}\right)_{\mathbf{R}^{m}} d t, \delta a\right)_{\mathbf{R}^{m}}+\alpha(\Phi(a-\bar{a}), \delta a)_{\mathbf{R}^{m}}
\end{aligned}
$$

which proves the theorem

Remark: Let us regard the special case of linear systems of ordinary differential equations (1.1) that attain the form

$$
\dot{x}(t)=B x(t)+g(t), \quad x(0)=x_{0} .
$$

Here, the $n^{2}$ elements $b_{i j}(i, j=1, \ldots, n)$ of the matrix $B$ are given functions of the unknown parameter vector $a \in \mathbf{R}^{m}$. Then the computation of $\operatorname{grad} J_{a}(a)$ requires

1. the solution of $(2.6)$ in order to find $x=x(t)$,

2. the solution of the adjoint equation

$$
-\dot{y}(t)=B^{*} y(t)+A(x(t)-z(t)), \quad y(T)=0
$$

in order to find $y=y(t)$ and

3. the computation of

$$
\operatorname{grad} J_{a}(a)=\sum_{i=1}^{m}\left(y, B_{i}^{\prime} x\right)_{z} e_{i}+\alpha \Phi(a-\bar{a}),
$$

where $\dot{B}_{i}^{\prime}=\partial B / \partial \check{a}_{i}$ can be found from $B$ by elementwise differentiation of $B$ with respect to $a_{i}$. 
Applying Theorem 1; any iteration step of the gradient method for minimizing (1.2)

$$
a^{k+1}=a^{k}-\gamma_{k} \operatorname{grad} J_{a}\left(a^{k}\right),
$$

requires the solution of one direct problem (1.1), and moreover the solution of one adjoint problen (2.2): Finally, formula (2.1) has to be verified. The parameter $\gamma_{k}$ in (2.7) denotes the step length parameter, which must be chosen appropriately. If $a^{k+1} \notin A_{\text {ad }}$, then an additional projection of $a^{k+1}$ into $A_{\text {ad }}$ is necessary.

Since such minimization problems (1:2) in general have a flat global minimum in deep banana-shaped valleys, problem (1.2) should better be treated by the GaussNewton method which makes it possible to proceed in great steps along the deep valleys and assures fast convergence. Let us denote by $G: a \in A_{\text {ad }} \rightarrow x \in X$ the implicit function for the system (1.1). Then in the Gauss-Newton method a given iterate $a^{k}$ is improved by

$$
a^{k+1} \stackrel{i}{=} a^{k}+\gamma_{k} \Delta a^{k}
$$

where $\Delta a^{k}$ is the solution of the linearized functional

$$
\left\|G\left(a^{k}\right)+G^{\prime}\left(a^{k}\right) \Delta a^{k}-z(t)\right\|_{z^{2}}+\alpha\left\|a^{k}+\Delta a^{k}-\bar{a}\right\|_{R^{m}}^{2} .
$$

Hence, (2.8) is given by

$$
a^{k+1}=a^{k}-\gamma_{k}\left(G^{*} \Lambda G^{\prime}+\alpha \Phi\right)^{-1} \operatorname{grad} J_{a}\left(a^{k}\right) .
$$

The computation of $G^{\prime}$ is the most time-consuming part of the Gauss-Newton iteration (2.9). One way to compute $G^{\prime}$ is the use of a finite difference approximation $G_{t}^{\prime}$ to the derivative $G^{\prime}$ :

$$
\begin{aligned}
& G_{\mathrm{r}}{ }^{\prime}(a)=\left(g^{1} \cdots g^{i} \cdots g^{m}\right), \\
& g^{i} \in Z, \quad g^{i}=\frac{G\left(a+\tau\left(a_{i}\right) e_{i}\right)-G(a)}{\tau\left(a_{i}\right)} \quad(i=1, \ldots, m), \\
& \left|\tau\left(a_{i}\right)\right|=\tau_{\mathrm{rel}}\left|a_{i}\right|+\tau_{\mathrm{abs}}, \quad \tau_{\mathrm{rel}}, \tau_{\mathrm{abs}}>0 .
\end{aligned}
$$

The derivative $G^{\prime}(a)$ can thus be approximated by solving the nonlinear, initial value problem (1.1) $(m+1)$ times.

The second way to compute $G^{\prime}(a)$ is the use of the Implicit Function Theorem. If (A 1) - (A 3) are fulfilled, then we have $G^{\prime}=-H_{x}{ }^{-1} H_{a}^{\prime}$. In this way there are no problems in choosing $\tau_{\mathrm{rel}}$ and $\tau_{\mathrm{abs}}$ appropriately and the amount of computational work is reduced. The route how to compute $G^{*} \Lambda G^{\prime}$ is given in the subsequent

Lemma 1: Let the assumptions (A 1)-(A3) be fulfilled. Then the (m,m)-matrix $G^{*} \Lambda G^{\prime}=\left(g_{\imath}\right)_{1}{ }^{m}$ can be computed by the following steps:

$$
\begin{aligned}
& \text { Solve } \dot{g}^{i}=F_{x}^{\prime} g^{i}+F_{a}^{\prime} \dot{e}_{i}, \quad g^{i}(0)=0 \quad(i=1,: ., m) \\
& \text { Compute } g_{i j}:=\int_{0}^{T}\left(g^{i}, A g^{i}\right)_{\mathbf{R}^{n}} d t .
\end{aligned}
$$

Remarks: 1. The $m$ initial value problems (2.10) are of the form (2.6). They are linear and can be solved simultaneously. 2. The gradient formula $(2.1)$ is equivalent to .

$$
\operatorname{grad} J_{\alpha}(a)=\sum_{i=1}^{m} e_{i} \int_{0}^{T}\left(g^{i}, \Lambda(x-z)\right)_{\mathbf{R}^{n}} d t+\alpha \Phi(a-\bar{a}),
$$

where the functions $g^{i}$ are solutions of $(2.10)$. 
Using Lemma 1 and Remark 2, one iteration step of the Gauss-Newton method (2.9) requires

(a). the solution of (1.1) in order to find $x=x(t)$,

(b) the simultaneous solution of the $m$ linear initial value problems $(2.10)$,

(c) . the computation of grad $J_{\alpha}^{\prime}(a)$ by formula (2.12),

(d) the generation of the matrix $G^{*} \Lambda G^{\prime}+\alpha \Phi$ using (2.11) and

(e) the computation of the new iterate $a_{\text {new }}:=a-\gamma\left(G^{* *} \Lambda G^{\prime}+\alpha \Phi\right)^{-1} \operatorname{grad} J_{a}(a)$.

\section{Parameter identification in a particular nonlinear initial value problem}

3.1 Mathematical model and properties. Let us consider a one-dimensional gasstorage model of aquifer type characterized by the space domain $[0, L] \subset \mathbf{R}^{1}$ :

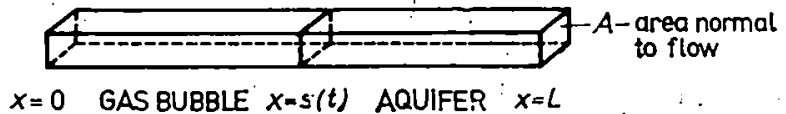

Fig. 1 One-dimensional flow model

We suppose that

(i) the permeability $k_{\mathrm{g}}(x)$ with respect to gas is much greater than the perme-

(ii) the water is incompressible and

(iii) for $t=0$ and for $x=L$ we have a constant pressure $p_{0}$.

Then the physical relations in flow of water and gas through porous media during a time interval $0 \leqq t \leqq T$ are characterized by

$$
\begin{array}{ll}
\frac{\partial}{\partial x}\left(a(x) \frac{\partial p_{\mathrm{w}}(x, t)}{\partial x}\right)=0 & (s(t)<x<L, 0 \leqq t \leqq T) \\
\eta A s(t) p_{\mathrm{g}}(t)=V(t) & (0 \leqq x \leqq s(t), 0 \leqq t \leqq T) \\
p_{\mathrm{g}}(t)=p_{\mathrm{w}}(x, t) & (x=s(t), 0 \leqq t \leqq T) \\
p_{\mathrm{w}}(x, t)=p_{0}>0 & (x=L ; 0 \leqq t \leqq T), \\
p_{\mathrm{g}}(t)=p_{0}>0 & (0 \leqq x \leqq s(0), t=0) \\
-a(x) \frac{\partial p_{\mathrm{w}}(x, t)}{\partial x}=c \dot{s}(t) & (x=s(t), 0 \leqq t \leqq T)
\end{array}
$$

where $a=k_{\mathrm{w}} / \eta_{\mathrm{w}}$ and

$\eta_{w}$ - water viscosity,

$p_{\mathrm{w}} \rightarrow$ pressure in the aquifer,

$p_{\mathrm{g}}-$ pressure in the gas bubble,

$\eta \quad$ - porosity of medium ( $\eta=$ const),

$V$ - amount of gas in the gas bubble under normal pressúre.

Equation (3.6) expresses that the water flow rate is proportional to the change of $s$ with respect to the time. An equivalent relation to (3.6) is given in the next Lemma: 
Lem ma 2: The equation (3.6) can be replaced by the equivalent condition

$$
p_{*}(s(t), t)=p_{0}+c \dot{s}(t) \int_{s(t)}^{L} \frac{d \xi}{a(\xi)}
$$

Proof: Owing to formula (3.1) partial integration yields for $p:=p_{\mathrm{w}}$ the relation

$$
0=\int_{\delta(t)}^{L}\left(a p_{x}\right)_{x} \cdot v d x=\left.a p_{x} v\right|_{\delta(t)} ^{L}-\left.p a v_{x}\right|_{\delta(t)} ^{L}+\int_{\delta(t)}^{L}\left(a v_{x}\right)_{x} p d x
$$

Now, setting $v=\int_{x}^{L} a(\xi)^{-1} d \xi$, we obtain in view of $v_{x}=-1 / a(x)$ and $\left(a v_{x}\right)_{x}=0$ the equation

$$
0=-a(s(t)) p_{x}(s(t) ; t) \int_{s(t)}^{L} \frac{d \xi}{a(\xi)}+p(L, t)-p(s(t), t)
$$

Using the boundary condition (3.4), formula (3.6) can be rewritten as (3.7). Thus, we confirm the assertion of the Lemma

From Lemma 2 we see that we can use either (3.6) or (3.7) in deriving properties of the Stefan problem (3.1)-(3.6). Throughout this discission we assume the existence of a classical solution $\left(s, p_{\mathrm{w}}\right)=\left(s(t), p_{\mathrm{w}}(x, t)\right) \in C^{1}[0, T] \times C^{2.0}\left((s(t), L),\left[0, T^{\top}\right]\right)$ to the Stefan problem (3.1)-(3.6). Furthermore, we will suppose that $L>s(t)$, $a(x) \geqq a_{0}>0$ and $c>0$.

Lem.ma 3: Let $\left(s_{1}, p_{w_{2}}\right)$ and $\left(s_{2}, p_{w_{3}}\right)$ be solutions to the Stefan problem. (3.1)-(3.6) corresponding to the data $V_{1}(t)$ and $V_{2}(t)(0 \leqq t \leqq T)$, respectively. If $0 \leqq V_{1}(t)<V_{2}(t)$ $(0 \leqq t \leqq T)$, then $s_{1}(t)<s_{2}(t)(0 \leqq t \leqq T)$.

Proof: Assume the contrary to the assertion and let $t_{0}>0$ be the smallest $t$ for which $s_{1}(t)=s_{2}(t)$. Then we have $\dot{s}_{1}\left(t_{0}\right) \geqq s_{2}\left(t_{0}\right)$. Now from $V_{1}\left(t_{0}\right)<V_{2}\left(t_{0}\right),(3.2)$ and (3.3) it follows that $p_{w_{1}}\left(s_{1}\left(t_{0}\right), t_{0}\right)<p_{w_{1}}\left(s_{2}\left(t_{0}\right), t_{0}\right)$. By using (3.7) we thus have

$$
p_{0}+c \dot{s}_{1}\left(t_{0}\right) \int_{\delta_{1}\left(t_{0}\right)}^{L} \frac{d \xi}{a(\xi)}<p_{0}+c \dot{s}_{2}\left(t_{0}\right) \int_{\theta_{1}\left(t_{0}\right)}^{\cdot L} \frac{d \xi}{a(\xi)}
$$

hence $s_{1}\left(t_{0}\right)<\dot{s}_{2}\left(t_{0}\right)$ in contradiction to $\dot{s}_{1}\left(t_{0}\right) \geqq \dot{s}_{2}\left(t_{0}\right)$

Lemma 4: Let $\left(s, p_{w}\right)$ and $\left(s^{s}, p_{w}{ }^{\delta}\right)$ be solutions to the Stefan problem $(3.1)-(3.6)$ corresponding to the data $V(t)$ and $V(t)+\delta(0 \leqq t \leqq T)$, respectively. If $\delta>0$, then

$$
s^{\delta}(t)^{2}<s(t)^{2}+\frac{\delta}{\eta A}\left\{\frac{2 t}{c}+\frac{\delta+2 V(0)}{\eta A p_{0}^{2}} \int_{s(t)}^{L} \frac{d \xi}{a(\xi)}\right\} / \int_{s \delta(t)}^{L} \frac{d \xi}{a(\xi)} .
$$

Proof: Multiplying (3.7) by $\eta A s(t)$ and using (3.2) yields

$$
V(t)=\eta A p_{0} s(t)+c \eta A s(t) s(t) \int_{s(t)}^{L} \frac{d \xi}{a(\xi)}
$$


Now integration provides

$$
\int_{0}^{t} V(\tau) d \tau=\eta A p_{0} \int_{0}^{t} s(\tau) d \tau+c \eta A \int_{s(0)}^{s(t)} x\left[\int_{0}^{L} \frac{d \xi}{a(\xi)}-\int_{0}^{x} \frac{d \xi}{a(\xi)}\right] d x .
$$

Therefore,

$$
\begin{aligned}
& \frac{c \eta A}{2} \int_{0}^{L} \frac{d \xi}{a(\xi)}\left[s(t)^{2}-s(0)^{2}\right] \\
& =\int_{0}^{t} V(\tau) d \tau-\eta A p_{0} \int_{0}^{t} s(\tau) d \tau+c \eta A \int_{s(0)}^{s(t)} x \int_{0}^{x} \frac{d \xi}{a(\xi)} d x
\end{aligned}
$$

Hence, the solutions $\left(s, p_{w}\right)$ and $\left(s^{\delta}, p_{w}^{\delta}\right)$ must both satisfy this equation. We subtract those versions and obtain

$$
\begin{aligned}
& \frac{c \eta A}{2} \int_{0}^{L} \frac{d \xi}{a(\xi)}\left[s^{\delta}(t)^{2}-s(t)^{2}\right]=\frac{c \eta A}{2} \int_{0}^{L} \frac{d \xi}{a(\xi)}\left[s^{\delta}(0)^{2}-s(0)^{2}\right] \\
& +\delta t-\eta A p_{0} \int_{0}^{t}\left[s^{\delta}(\tau)-s(\tau)\right] d \tau+c \eta A \int_{\delta(t)}^{\delta^{\delta}(t)} x \int_{0}^{x} \frac{d \xi}{a(\xi)} d x \\
& -c \eta A \int_{s(0)}^{s^{\delta}(0)} x \int_{0}^{x} \frac{d \xi}{a(\xi)} d x
\end{aligned}
$$

where we have used the property

$$
\int_{\delta_{\delta}(0)}^{\delta^{\delta}(t)} \varphi(x) d x-\int_{\delta(0)}^{\delta(t)} \varphi(x) d x=\int_{\delta(t)}^{\delta^{\delta}(t)} \varphi(x) d x-\int_{s(0)}^{s^{\delta}(0)} \varphi(x) d x, \quad \varphi(x)=x \int_{0}^{x} \frac{d \xi}{a(\xi)} .
$$

From Lemma 3 we have learned that $s^{\delta}(t)>s(t)$, hence

$$
\begin{aligned}
& \int_{0}^{L} \frac{d \xi}{a(\xi)}\left[s^{\delta}(t)^{2}-s(t)^{2}\right] \\
& <\int_{0}^{L} \frac{d \xi}{a(\xi)}\left[s^{\delta}(0)^{2}-s(0)^{2}\right]+\frac{2 \delta t}{c \eta A}+2 \int_{s(t)}^{\delta^{\delta}(t)} x \int_{0}^{x} \frac{d \xi}{a(\xi)} d x-2 \int_{s(0)}^{\delta^{\delta}(0)} x \int_{0}^{x} \frac{d \xi}{a(\xi)} d x .
\end{aligned}
$$

Using again $s^{d}(t)>s(t)$ we find

$$
\int_{0}^{s(t)} \frac{d \xi}{a(\xi)} \leqq \int_{0}^{x} \frac{d \xi}{a(\xi)} \leqq \int_{0}^{s^{\delta}(t)} \frac{d \xi}{a(\xi)} \ldots \text { for } \quad x \in\left[s(t), s^{\delta}(t)\right]
$$


Therefore we have

$$
\begin{aligned}
& \int_{0}^{L} \frac{d \xi}{a(\xi)}\left[s^{\delta}(t)^{2}-s(t)^{2}\right] \\
& <\int_{s(t)}^{L} \frac{d \xi}{a(\xi)}\left[s^{\delta}(0)^{2}-s(0)^{2}\right]+\frac{2 \delta t}{c \eta A}+\int_{0}^{s^{\delta}(t)} \frac{d \xi}{a(\xi)}\left[s^{\delta}(t)^{2}-s(t)^{2}\right]
\end{aligned}
$$

$=$ which yields the expected inéquality ${ }^{\prime}$.

Now we are able to prove a more general monotonicity. theorem.

Theorem 2: Let $\left(s_{1}, p_{w_{1}}\right)$ and $\left(s_{2} ; p_{w_{2}}\right)$ be solutions to the Stefan problem $(3.1)-(3.6)$ corresponding to the data $V_{1}(t)$ and $V_{2}(t)(0 \leqq t \leqq T)$; respectively. If $0 \leqq V_{1}(t) \leqq V_{2}(t)$, then $s_{1}(t) \leqq s_{2}(t)(0 \leqq t \leqq T)$.

Proof: For $\delta>0$, let $\left(s_{2}{ }^{\delta}, p_{w_{i}}^{\delta}\right)$ be solutions to the Stefan problem (3.1)-(3.6) corresponding to the data $V_{2}(t)+\delta$. From Lemma .3 we see that $s_{1} \leqslant s_{2}{ }^{\delta}$. Using Lemma 4 we find

$$
s_{1}(t)^{2}<s_{2}(t)^{2}+\frac{\delta}{\eta A}\left\{\frac{2 t}{c}+\frac{\delta+2 V_{2}(0)}{\eta A p_{0}^{2}} \int_{s_{2}(t)}^{L} \frac{d \xi}{a(\xi)}\right\} / \int_{s_{2}}^{L} \frac{d \xi}{a(\xi)}
$$

Since $\delta>0$ can be chosen as small as desired, it follows that $s_{1}(t) \leqq s_{2}(t)$

As a corollary of Theorem 2 we obtain the following uniqueness theorem.

Theorem 3: The solution $\left(s, p_{w}\right)$ to the Stefan problem $(3.1)-(3.6)$ is uniquely determined.

Proof: If $\left(s_{1}, p_{w_{1}}\right)$ and $\left(s_{2}, p_{w_{2}}\right)$ both correspond to the data $V(t), 0 \leqq t \leqq T$, then from Theorem 2 we have $s_{1} \leqq s_{2}$ and $s_{2} \leqq s_{1}$, which implies that $s_{1}=s_{2}$. Therefore we have $\dot{s}_{1}=\dot{s}_{2}$. Now using (3.7) we obtain $p_{w_{1}}\left(s_{1}(t), t\right)=p_{w_{1}}\left(s_{1}(t), t\right)(0 \leqq t \leqq T)$. Owing to (3.4) and (3.1) this provides $p_{\mathrm{w}_{1}}=p_{\mathrm{w},}$

3.2 The identification problem. The identification of the unknown function $a=a(x)$, $0 \leqq x \leqq L$, from measured field data values $V(t)$ and $p_{g}(t)(0 \leqq t \leqq T)$ is of great practical importance. In order to realize this aim it suffices to consider the free bound-. ary $x=s(t)$. Owing to (3.2), (3.3) and (3.7) we have

$$
p_{o}(t)=p_{0}+c \dot{s}(t) \int_{s(1)}^{L} \frac{d \xi}{a(\xi)} \quad(0 \leqq t \leqq T)
$$

We assume that there are given functions $\varphi_{i}, \varphi_{i}(x)>0(0 \leqq x \leqq L ; i=1,2, \ldots, m)$ such that

$$
(a(x))^{-1}=\sum_{i=1}^{m} a_{i} \varphi_{i}(x) \quad\left(a_{i} \geqq 0, i=1,2, \ldots, m\right) .
$$

Moreover, let us suppose that the antiderivatives of $\varphi_{i}$ possess a simple structure such that $\psi_{i}(\tau)=\int_{r}^{L} \varphi_{i}(\xi) d \xi(0 \leqq \tau \leqq L ; i=1,2,:: ;, m)$ is explicitly available. Then (3.8) 
may be rewritten as

$$
p_{0}(t) \stackrel{i}{=} p_{0}+c \dot{s}(t) \sum_{i=1}^{\dot{m}} a_{i} \psi_{i}(s(t)) \quad(0 \leqq t \leqq T)
$$

For given positive values $\eta, A, p_{0}, v_{0}=V(0)$ the initial value $s(0)=s_{0}=v_{0} /\left(p_{0} \eta A\right)$ is determined. Thus, the initial value problem

$$
\dot{s}(t)=f(t, s, a):=\frac{p_{g}(t)-p_{0}}{c \sum_{i=1}^{m} a_{i} \psi_{i}(s(l))}, \quad s(0)=s_{0}
$$

attains the form (1.1) with $n=1$. The noisy observations $z(t)=s(t)+\delta(t)(0 \leqq t \leqq T)$ are won from noisy. measurements $z_{1}(t)=V(t)+\delta_{1}(t)(0 \leqq t \leqq T)$ and $z_{2}(t)=p_{\mathrm{g}}(t)$ $+\delta_{2}(t)(0 \leqq i \leqq T)$ by the formula (cf. (3:2)) $z(t)=z_{1}(t) /\left(\eta A z_{2}(t)\right)$. When a fixed vector $a \in A_{\text {ad }}=\left\{\tilde{a} \in \mathbf{R}^{m}: \tilde{a}_{i} \geqq 0(i=1,2, \ldots, m)\right\}$ is considered, then in view of Theorem 3 the direct problem (3.9) is uniquely solvable. On the other hand, the inverse problem of estimating $a \in A_{\text {ad }}$ from the data $z$ may be realized by regularized parameter identification (see (1.2)). Then

$$
J_{\alpha}(a)=\frac{1}{2}\|s-z\|_{L,\{(T)}^{2}+\frac{\alpha}{2}\|a-\bar{a}\|_{\mathbf{R}^{m}}^{2}
$$

is to be minimized over $a \in A_{\mathrm{ad}}$. However, the solutions $s=s_{a}$ of (3.9) are in practice also perturbed. Namely, the values $f(t, s, a)$ must be replaced by $\tilde{f}(t, s, a)=\left(z_{2}(t)-p_{0}\right) \mid$ $\left(c \sum_{i=1}^{m} a_{i} \psi_{i}(s(t))\right)$. Thus, this additional error is to be taken into consideration when a discrepancy principle for choosing the regularization parameter $\alpha>0$ is used.

Acknowledgement. The author is grateful to Prof. Dr. B. Hofmann for several remarks and constructive comments that contributed to the finished form of the paper.

\section{REFERENCES}

[1] Bock, H. G.: Recent Advances in Parameteridentification Techniques for O. D. E. (Preprint no. 567). Univ. Bonn 1982.

[2] Friedrich, V., Hofmann, B., und U. Tautenhahs: Möglichkeiten der Regularisierung bei der Auswertung von Meßdaten. Wiss. Schriftenr. Techn. Hochsch. Karl-Marx-Stadt 10 (1979), $1-99$.

[3] Hofmann, B.: Regularization for Applied Inverse and Ill-Posed Problems ('TeubnerTexte zur Mathematik: Bd. 85). Leipzig: B. G. 'Teubner Verlagsges. 1986.

[4] Hormann, B.: On the Analysis of a Particular Volterra-Stieltjes Convolution Integral Equation. Z. Anal. Anw. 7 (1988), 247-257.

[5] Hofmann, B.: On a particular initial value problem with. an application in reservoir analysis. Indian J. Pure Appl. Math. (submitted).

[6] Nowak, U, and P. Dedflyard: Towards Parameter Identification for Large Chemical Reaction Systems. In: Numericil Treatment of Inverse Problems in Differential and Integral Eqúations. Proc. Conf. Heidelberg (Fed. Rep. of Germany), 30.08.-3.9. 1982 (Ed.: P. Deuflhard and E. Hairer). Boston-Basel-Stuttgart: Birkhüuser Verlag 1983. p. $13-26$.

[7] Tautenhage, U.: Parameter identification from distributed observations. Preprint: Karl-Marx-Stadt: Techn. Univ., Preprint Nr. 25 (1987). 
[8] Тихонов, А. Н., и В. Я. АРсенин: Методы решения некорректных аадач. Москва: Изд-во Наука 1979.

[9] Yermakova, A., VAJda, S., and P. Valko: Direct integral method via spline approximation for estimating rate constants: Applied Catalysis 2 (1982), 139-154.

[10] ZrIDLER, E.: Vorlesungen über nichtlineare Funktionalanalysis I. Fixpunktsätze (Teubner Texte zur Mathematik, Bd. 2): Leipzig: B. G. Teubner Verlagsges. 1986.

Manuskripteingang: 24. 11. 1987

\section{VERFASSER:}

\section{Dr. Ulrich TautenhahN}

Sektion Mathematik der Technischen Universität

PSF 964

DDR -9010 Karl-Marx-Stadt 